\title{
Un Expediente Inédito de Rodriguez de Mendoza
}

Dentro de los manuecritos de velor, teizmento salvados de! meendio

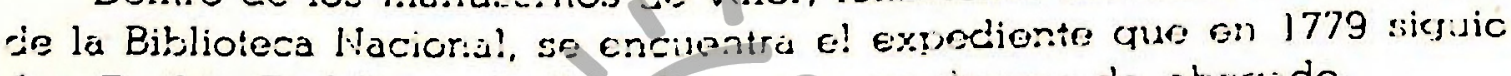
don Toribio Rociriquez de Mendczis rata craciuarse de abogado.

El expediente al parece: completo, eonsta de nueve hojas hoy sueltes. con todos los borcies quemudos y muchas de ellas casi liergibies cebidio a la acción del equa utilizaja para combatir a aquel "opílono lógico del largo calvario de la inteligencita en que hace muchos años vive el Perü", corno el Dr. Pcrras Bartenechiea liamé coir gran propie ded, al infausto siniesiro del 10 de rnùyo de 1943.

El interés histórico dêbexpedirnte rádica en la calidad de las piozis que b iniegran, las gue sin duda hacen dars luz sobre cierlos aspectos de lé persona del iluelre Redfofldel Convidotícle San Carlos.

Aparte de la pueba fehaciente sobre los grodics do Bachiller enSegrados Cánones y de Abogado que Rodrígiez de Mendoza opló, se encuentra en el referido expediente, preciosos documentos que atañon directamente a su personá, tales como la partida de bautismo, lá legitimidad y pureza de sangre suya propia y la de sus inmediatos ùntepasados.

Diríase que se trata de documentos seguramente descoriocidos por los biógrafos de Rodriguez de Mendoza, si se tiene on cuenta que ninguno de ellos los citi concretamente. Jorge Guillermo Leguía on si: sugestivo ensayo titulado "El Precursor" repite aquello que refiero don José Toribio Polo, de ser Rodríguez de Mendoza "hijo de cristianos viejos, y de solar conocido"... carente de toda "mezcla con sangre de moro...", etc. Pero ni estas referencias del nombrado investigador poruano, ni aquella repetición de nuestro acucioso historiógrado Leguía, prematuramente fallecido, se hallan respaldadas por la cita del docu- 
mento respectivo. Ello nos induce a suponer que ambos desconocieron el expediente que hoy publicamos. Además, corrobora tal supuesto, el hecho de que según la partida de bautismo que ofrece el propio Rodríguez de Mendoza, aparece que él nó nació el 15 de abril de 1750 - fecha hasta hoy generalmente aceptada-, sino que debió haber nacido el 17 de ese mes y año; pues, si hubiese sido utilizado este documento ya sea por los nombrados biófragos del Precursor, o por sus posteriores exégetãs, alguien, sin duda, hakria reparado en esta circunstancia ciertamente fundamental.

\section{Mlejardro HERNANDEZ ROELEDO.}

Lima, Abril de 1950.

\section{P. S.}

(FG 1.) El D.D. Toribio Fodrigus: de Meridoza en la mejor for/ma ơ. haya lugar en arp paresco ante V.A. Y digo: $\sigma^{*}$ h:Di/encome dodicado al estudio do la Jurisprudencia $p^{\not}$ en ella lo/grar el tifulo de Ábogado. teniendo conciuidas lás staciones (sic) $q^{*}$./ 1\% ello me habilitan segun lo esclarecen las certificaciones del Grado de Bachiller, y la del $A$ bogado ante quien he cracti/cado el toro $c^{\prime \prime}$. acompañadas con lis partida cie Bautismo./y inlormacion de mi limpiesa de sangre, con la dehida so/lemnidad presento: para lograr el efecto de mis deseos me / resta el $q^{c}$. la justiifcacion de $V$.A.me admita a la pruebi de / Abogado acostumbrada: por tanto

A V.A.pido q.habiendo $p^{r}$.presentadas las certificaciones $t e$ informacion roferidas en su consequencia se sirva ad/mitirme a la prueba de Abcoado a $q^{\prime \prime}$. estoy liano, pues en ello / procedera segun "la eçuidad. y Gusticia a". pido \&.7 ISO"

(Fdo.) D. Toribio Rodriguez / de Mendoza. (Rúbrica).

(Fa 1 v.) (Nola al murgen) Srs.Bravo / e! Conde / Manzilla, / Rivera./

Vista al Señor Ministro q.hacia / der Fiscal. Lima y Moyo velnie do / mil selecientos setonta y nuebe./

(Hay dos rúbricas)

(Fdo.) Martin de Pro. (fiúbrica).

M. P. Sor.

E1 Oider fiscal on vista de este expedt". Di/ce: Que pr.los docum\%s. q.presenla el $/$ dr.dn.Toribio fodrigz. hace constar todos los requisitos de la ley pi. poder ser / admitido al.examen de Ábogado acoslum/brado, pr.lo q.le parece al Fiscal que / V.A. podra mandarlo assi. c resolver / lo q.mas sou de Justq. Lima y Mayo 21 / de $1779 . /$

(Fdo.) El Marq".de Corpa. (Rúbrica.) 


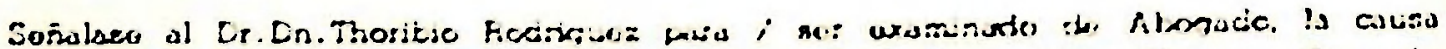

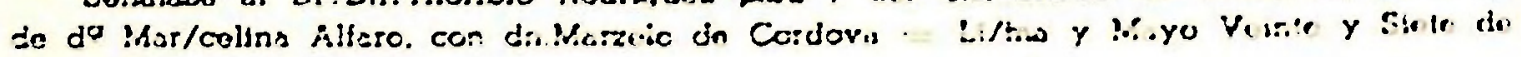

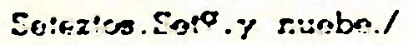

(Hay un signo y usa ribsico.)

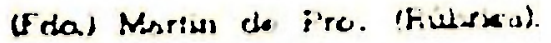

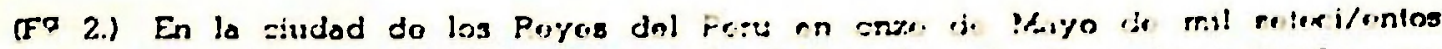

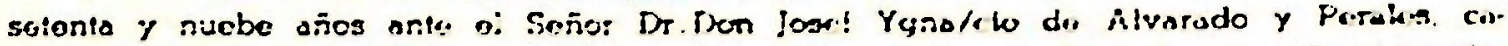

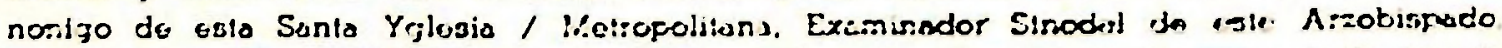
P:o/risco: y Viearic col Monastotio de Sants Cotholins Jur.z / dir Liesmon y ficctor de

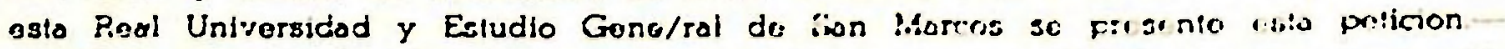

פ Dxtc: Don Thoribto Rodriguez. Precbilato corro nas / hats lisgu: parcesco anto

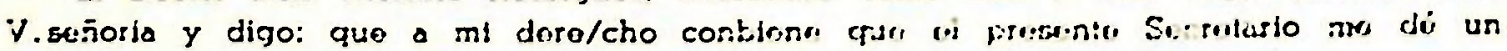

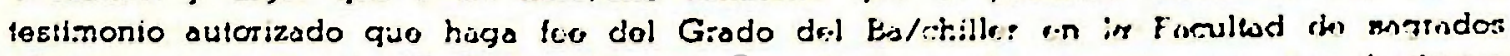

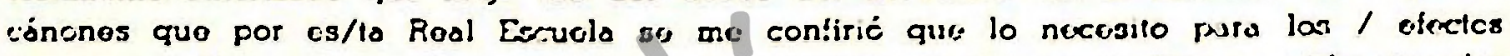
que mo coabongan on culo otencien $=A$.vuosanch / pido. $Y$ suplino 30 sirba mandis:

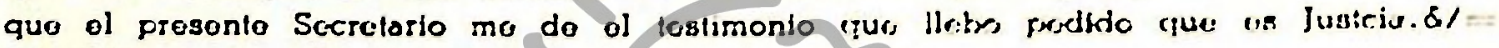
(Fdo.) Doctor Don Thoribio Rodriguoz.

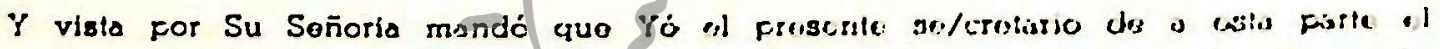

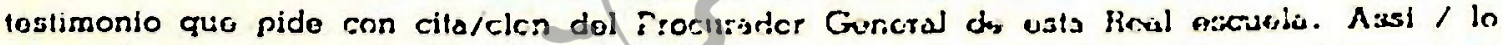

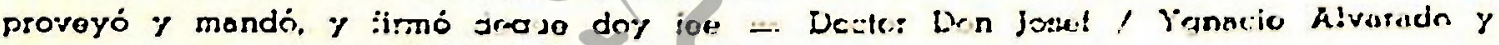
Perales = Dn. Bernate Cor!!ja de / Bibar Strreturio.

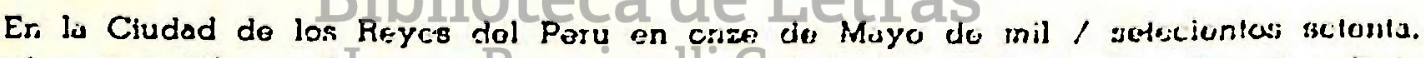

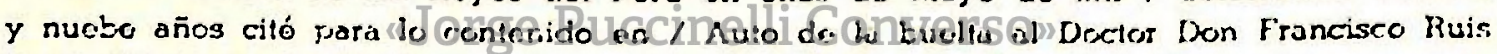

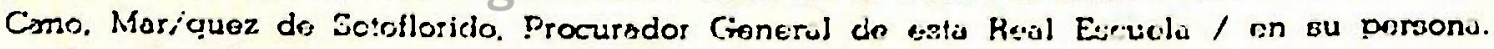
= Doy foe. Don Bernute Cor!lio y Bibar. Sereretario.

En exectucion y cumplimtr. de lo pedido y mundudo en el oscrito / y Auto $\tau^{n}$. intecondr

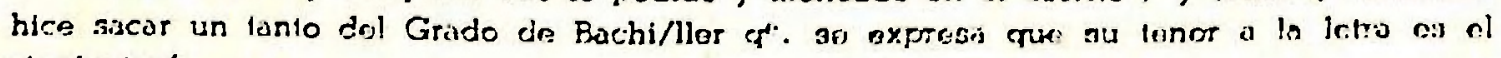
siruiente:/

(Fa 2 v.) En la Cludad de loz Royes del Peru on die\% y sois do Arril do mil setel cienlos selenta y nuobe años, viernes a las deaz dol dia mus / o munos, on al Gonoral Maior de esta Real Universidad y Estudic / Gonoral de San Marcon anto mi ol Secrulario y testigos, el Señor Doctor Don Josel do Baquijano dió y confirió el Grí/do do Buchi. ller en la Focultad de Sagrados Cánones al Doc/tor Don Thoribio Rodriguez habiencio procedido Ynlormacion / de háher consluldo ins Cursos quo prescriben los Estututos / do está Real Escuela y el stclo Publico de Conclusiones y Ar/gumentos y los demás requeistos de Solemridad acoslumbra/dos conforme a los conslituciones de esta Real Uniberci/dad estando presenies el Señor Doctor Dor Josel Ygnacio / do Alvarado, y Porales Canonigo de esta Santa Yglesía Me/tropolitana. examinador eynodal de esto Arzoblosia- 
(lo / Provisar y Vilirio dol Monasterio de Santa Cathalina / Examinador Synodal do esto Arzobispado. (ste) fLoz do Dioz/mos y. Rector de esta dha. Unibercidad. y algun numero do / Doctores, stondo testigos los vedalos de ctha. Unibercidid, $y$ otras unuchas personas do que doy tee $=$ Dan Bermibe Cor/tijo de Bibar, Secretario.-

Sergun parece de los fiulos que sa formaron paira dho.grado. y a la letra dice b / ge. va insorto a qe.me romito y fasa qe. conste doy el presente en la ciudad / Ie los Royos del Peru on doco de Mayo de mil selecientos setenta y nuebe años./

Bemabe Cortljo da Vibar / Secrelario.

(F" 3.) El Dacter Don Tortbio Rodriguez do Mendoza vecino de la ciu/dad de Chachepoyos $y$ resiciontey on esta Capital en la / mejor forma cuo haya lugar en Dro.parezco nrio / V.S. Y digo: Quo al mlo convlene se me reciba Ynforma/cion acerca de la lexitinildad $y$ natules do mi Porsona $y / q^{e}$. los Testigos que presentare so examinen por el te/no: de oste y $\mathbf{q}^{*}$. expresen cómo os cierto, que soy na/tural de aquella Ciudid.

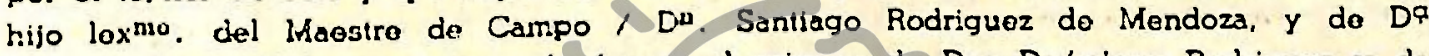
Maria / josola Collanies quienes lo fueron el primero de Don Do/mingo Rodriguez $Y$ de DQ Angela Emani de Arbil/do, y la eogunda de Du. Josel Fabian Collantes, y de Da Rosa Garcia de Perea, havidos todos y repulados en aquel/vecindarto pr. Gente de la primera diotincion, y como tales/han exercitado los empleos qe. gradúan esta qualidad i y concluida q". sea tha. Ynforun. se me entregue ori/ginal pa los electos q. me convoncja: Por tanto $=/$ a V.S. pido, y supco. que con Cltasn. del Procuror. Gral. de la Ciudad so mo reciba la Ynfomasov. $q^{*}$. ofrezco, y / concluida $q^{\prime \prime}$. sez se me entregue originel pi el / uso de mi Dro.p en ollo proceder segtin justa./pido \&.

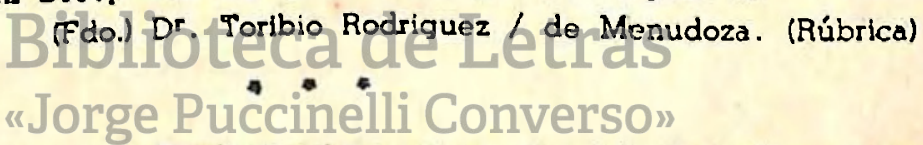

(A) margon superior derochol: Recibase / en esta parte / la Ynfor/macion a.ofrece / con ostacion / del Procurador / Gen'. do la Ciudad.

(Rúbrica)

(Fa 3 v.) En la Ciuriad de los Reyos dol Peru en siele de Mayo / de mil seteclentos setenta y nuobs años ante el Sos. Nísstro de Campo Don Ysidro de Abarca. Caballero / do la Orden do Santiago. Alcalde Ordinario / de Cola Ciudd. y su Jurisdiccion pr. S.M. so prosentó / esta Pelicion.

Y vista por Su Señoria mandó se reciba a esta / parte la Ynformacion que ofrece con citacion del / Procurador gral. de la Ciudad y asi lo proveyó y / firmó con parecer del Liconzdo. $\mathrm{d}^{\mathrm{n}}$. Cayetano Velon, Abogilo. de esta $\mathrm{R}^{\mathbf{l}}$. Audiencla, $y$ Asesor nombrado / de orn.de los soñores Alcaldes. Ante mi Josseph de Aiscorbe./ Escno. de S.M. y Puco.

Yasdro (Rúbrica.) 


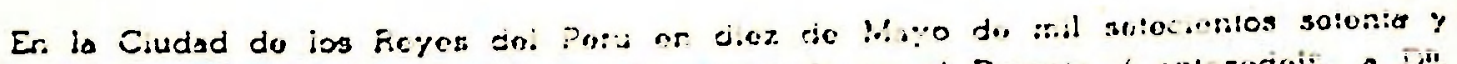



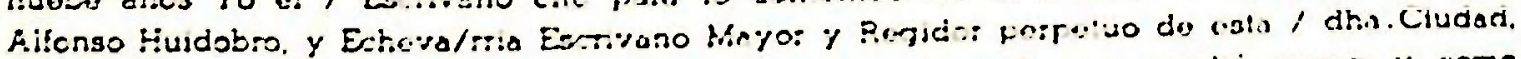

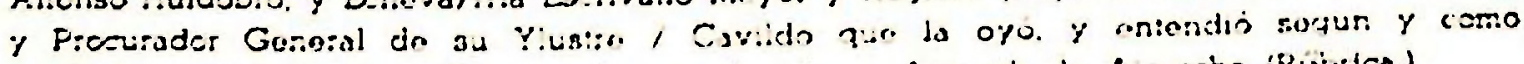

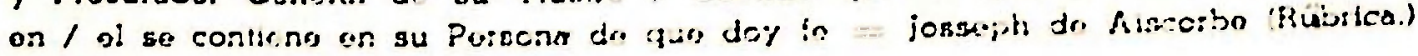

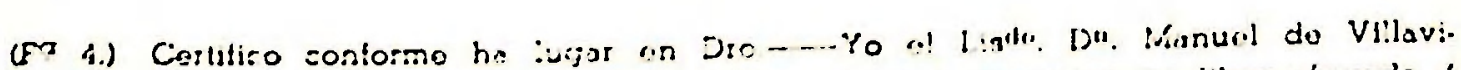

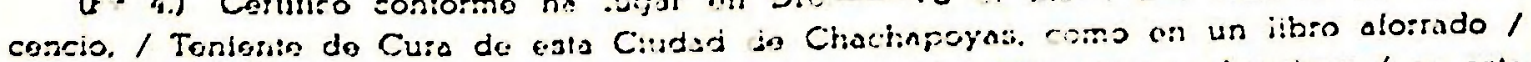

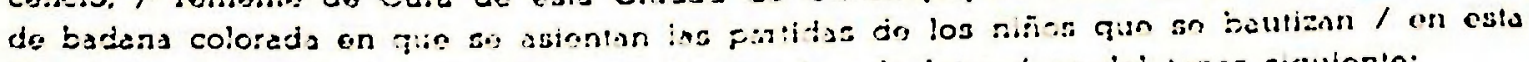

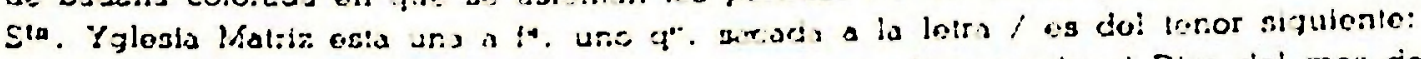

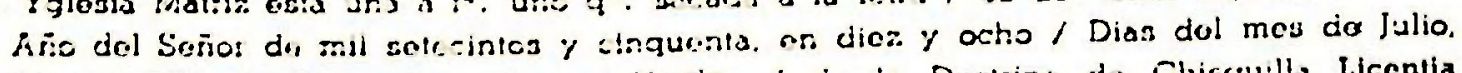

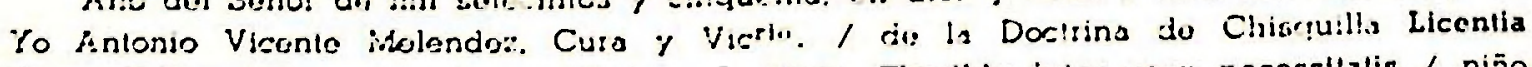
Parroqu! Eaupilsci. pu/su oleo y Chrisms a Alojo. Thoribio inter carg nocessitalis / niño

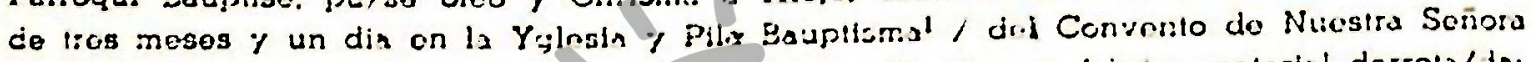

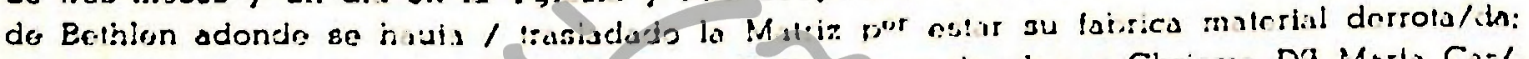

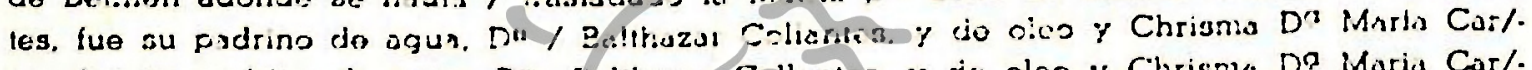



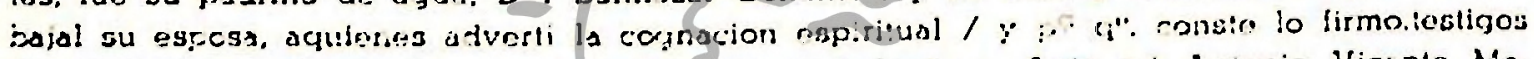

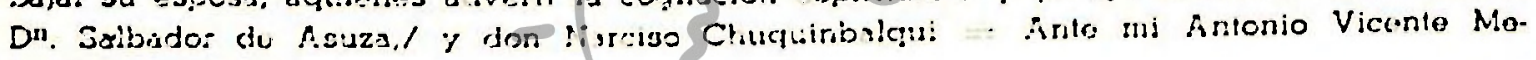
lender $=:$

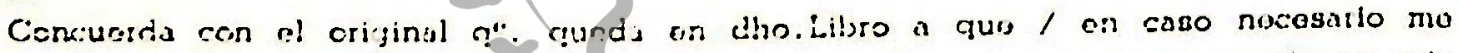

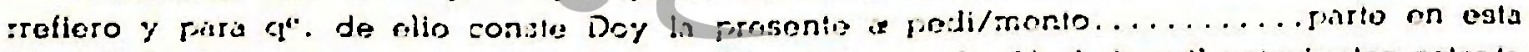
Ciudad de Chachepoyas on veinto / cinco Dias dol ines do hbril do inil selecientos sotonta i osho ar̃os/.

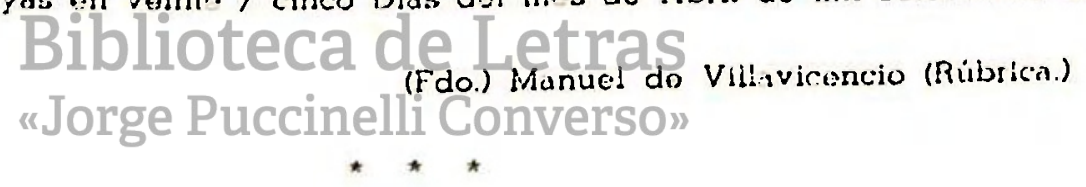

(Fa 5) (Al margen) Testigo / Dn. Jese En/lonio More/no Truxillo / $N$. G. de mas/ 60 años de edad./

En la Ciudad de los Reyes del Perú en diez de Mayo / de mil selecientos solonta, y nuobe años. El Dr. Dn. Toribio Ro/drlguez porá la Yn!ormain. que tione ofrecida, y le está / mandada dar presenió por testicjo a Don Jose Anto/nio Moreno Truxillo a quien rocibi juramtio. quo lo / hizo por Dioz Nro. Sur. $i$ a lina Ciuz bajo del qual/ prometló decir verdad. y siendo preguntado al tenor / dol Escrito presentado = dijo: que con acasion do ser / el Testizo notural do le ciucied de Moyobamba en / la Provincia do Chachapoyas sabe, y lo consta que el / Dr. Dn. Toribio Rodriguez q". lo presenta es natural / de la Ciudad de Chachapcyas, hijo Lexitimo de $D^{n}$./ Santiago Rodriguez, y de D' Moria Jossefa Collan/tes aquienes bió casar el Testigo. Que el dho. Mre. do Campo Dn. Santiago Rodriguez de Menclozá es hilio Legílimo de $\mathrm{D}^{u}$. Domingo Rodriguez y de Dạ. An/gela Erriani de Arbildo. Que Dr. Maria Jossefa Co/llantes es hija Logítima de $D^{n}$. Jo/se Fabian Co/llantes, y de Da. Rosa Garcia Perea. Que todos los re/feridos han eido tenicios y rerutados en acuella Ciu/dad y Prov. por Personas de la primera distins ${ }^{\circ n}$. y / Nobleza, y $q^{e}$. como tales han efercido los Empleos ho/noríficos $q^{e}$. en aquella Ciudad se confieren a las / personas de distincion : Que osto que lleba dho./y 
do si ns púbises y neseric pibisca voz. y fana, y la verdad vajo del juramto. / que tlene ancho en el q". se air/mó y ratlitió siendole leida quo no le tocan las Gane/rales do ia Ley. q". es do hexiad de mís de exsanta años $/ y$ io firnó de c". doy Fe = José Anonio / ilorono Truxillo./ Anto ml' (Fjo.) José de Aiscorive.;

(Fa' 5 r.) Manl. (oseph do Nieneses Contador Reguladicr de ios Ra.dros.de / Media Aunatis y Lunzas de esie I"nu. Cartifico: que en el libro manual que al / presente corre an la Conisdf do mi cargo, a 4.40 bla. do el so halla sentada una parti/da de! tenor siguionto-...

En ceho do junio de mil solocienios setenta y nue/be años pagó en la Rl. Caxa de Lirra, el Dr. Dn./ Thoribio hodriguez Nueve $p^{s}$. y scis ${ }^{\mathrm{N}}$. on do/bles (en que se incluye la condon a Lipa a diex / ccho por ciento) que pertonecia a! Rl. dro. de Kodia / Annata, ecr el honor del examna quo obtubo de / Abogsdo de esta $R^{1}$. Audp. son el Rl. Acuerdo cial/ Justicia donde fluo aprobado segun consta de la Cer/ition. dada for D"l. Martin Pro Escnn. de Carmaro de òha. $\mathbb{R}^{1}$. Aude. de sieto dho.mes; cuio / entoro se hizo por to gulacion del Contor. de oste d:o./ del cilado dia siete que aprokó el Sr. Jugz en Villete de la misma tha. como consta de el, recibo / puesto a su continuacion de los Ofiz'. $\mathrm{R}^{\circ}$ a quie/nes se hace cargo de dho". p*.......009 pesos 6 reales./

Sogun consta do dicho libro foxa y partida a que me refiero, y para que conste doy la / presente en los Reyes del Perú en dho.dia mes y año. (Fdo.) Manuel Joseph de Mienesos.

(F⿳ 6.) Otroi Dil. Franiu. del los Santos/ Barba N. G. de 45 as.

En la Ciudad en el dia "Mos. y año releridos parala ex/prosada Ynformas". presentó por Tğo. a Dn. Franco. de / los Santos Earka vecyno, y del comercio de esta ciudad / de quien recibi juramto. que lo hizo por Dios Nro. Sr./ a una Cruz segun forma do dro. vajo del gual prometio / decir verdad, y siendo preguntado al tenor del escrito pre/sentado $=$ Dijo que con ocasion do la amistad que profesó / con Dn. Josse Fabian Collantes sabe $y$ le consta $q^{*}$. el dho./Dn. Toribio Rodriguez $q^{\mathbf{e}}$. le presenta es nalural de la Ciu/dad de Chachapoyas, hijo legítimo de Dn. Santiago Rodri/guez de Mendoza aquien conoció en esta ciudad, $y$ de Dọ/ Maria Josséa Collantes su legítima muger. Y el / dho. Dn. Santiago es hijo Legitimo de $D^{n}$. Domingo Ro/driguez y de Doña Angela Ennani de Arisildo. que la / dha. Da Maria Jossela Collantes es hija de $D^{n}$./ Jose Fabian de Collantes. y do Dạ Rosa Garcia de Peres / todos cristianos viejos limpios de loda mala raza, perso/nas de distinguida Nobleza $q^{\prime \prime}$. como tales han ejercido en $/$ aquella ciudad los empleos honorificos de republica $q^{\prime}$. con/liere su cavildo a las Personas de lustre. Quo esto $q^{e} /$ bu dho. y declarado es la verdad vajo de juramin $q^{e}$. trae / hocho on el $q^{c}$. Se afimnó. y ratificó siondole leida $q^{e} . /$ no le tocan las Generales de la Loy, y $q^{n}$. es do odad de cuaren/ta y cinco años y la firmó de que doy fe = (Fdo.) Franıo. do los Santos / Barba (Rúbrica.) Ante mi = Josseph de Aizcorbe. 


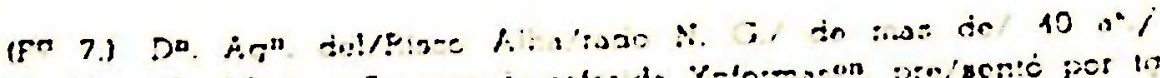

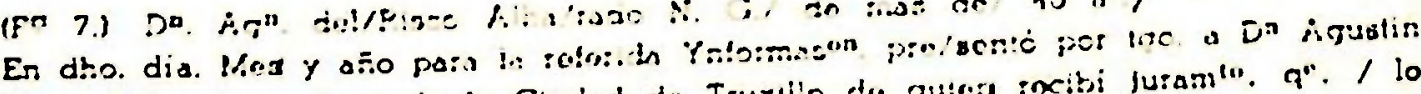

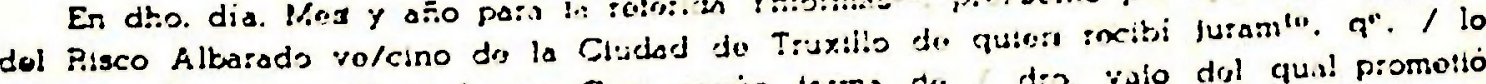

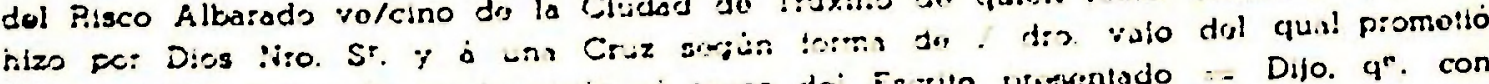

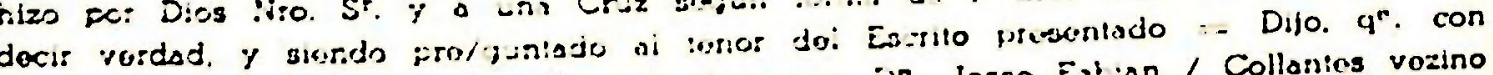


to is Ciudad de Chercharoyas aize $y$ lo consis " $"$ ". Dn. Thoriblo Rodriguoz a". lo pro.

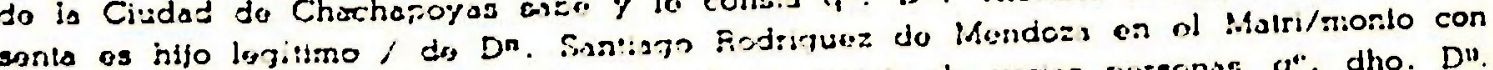

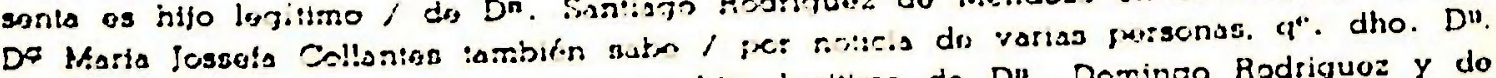

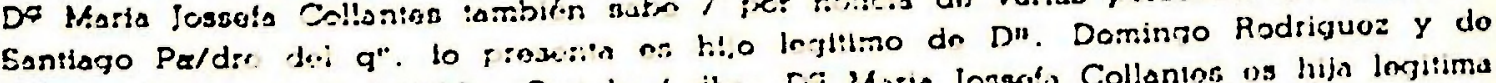

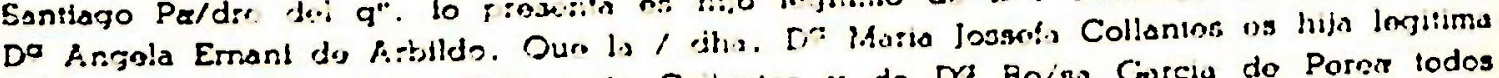

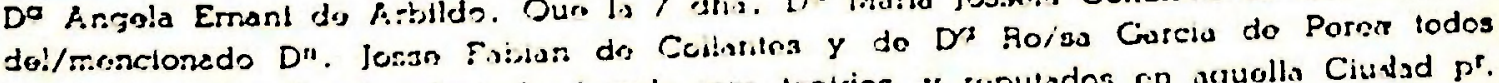

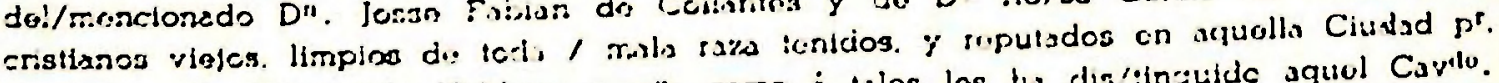



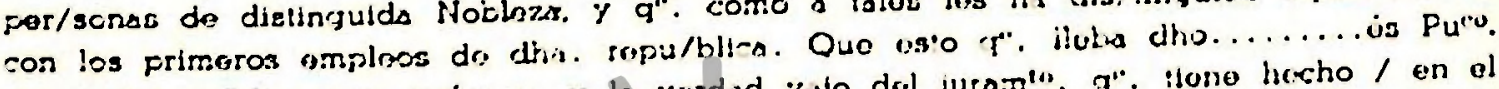

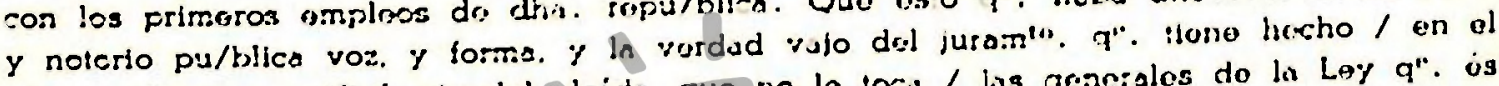
$q^{*}$. so afirmó $y$ ratlifico. siondo!e loida, qun no le locis / has grancolos do la Ley q". is

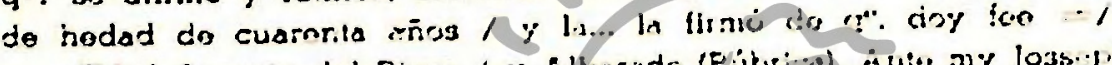

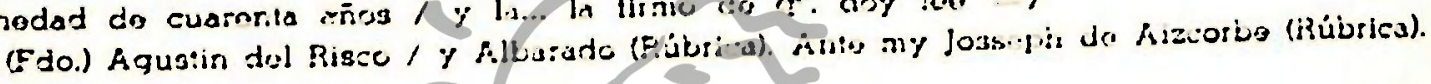

(F) 7 v.) En blanco.

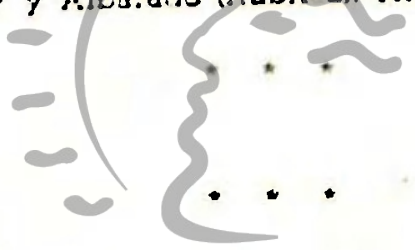

(F' 3.) ....... Manuel Jph. do Pasqual Herasso, Abogido / de la Rl. Audq. ó Ceritico on quanto pueso. Y haclugar en 2 dre. Cas el Dr. Dn. Thoribio Rod:igx. ho concu/rrido y trectontado dlarlami" mi ostudio f a practicar, ó instruirse en las ma. terias / del foro: lo $q^{\prime}$. ha conseguido a oxfuorses / do su uplicas". hasta llogar à aptitud de / poder ser recibido por Abogado on ssta / $\mathrm{R}^{\prime}$. A.udq y $\mathrm{p}^{\alpha} \mathrm{q}^{\prime \prime}$. obre los eloctos $q^{*}$. lo convon/gan al interesado doy esta en Lima a / 19 de Mayo de 1779.

(Fdo.) Man'. Jph. de Horasss. (Rúbrica.)

(Fa 9.) (Al margen supericr derecho).

Sres. / Regente / Bravo / Conde / Mansa / Ribera / Muta Linaros./

Por aprobado, y entro a Jurar. Lima y Junio / cinco do mil seleclontos selenta y nuebo./ (Trog rúbricas diferentes.)

(Fdo.) Martín de Pro. (Rúbrica.)

Cortifico en conlomidad del Auto que ante/cede el Dron. Thorivio Rodriguoz; hizo el juramento / acostumbrado, de usar bien y fielmente el cargo do / Abogado do osta Real Audiencia sin llevar derechos / injustos, ninguno a los Pobres ni all Real Fisco; dofon/der la Pureza de la Virgen Marla; y aquellas que / segun su estado lo son permitidas por la Loy y / para que conste doy la presente, en los Reyes / del Perú en cinco de Junio de mil Selecientos selen/la y nuebe an.

(Fdo.) Marlín de Pro. (Rúbrica.)

(Fa 9 v.) En blanco. 